\title{
Il popolamento a Orthoptera e Mantodea del Parco Regionale del Corno alle Scale (Appennino Tosco-Emiliano): dati bibliografici e nuove segnalazioni di elementi boreo-orofili (Insecta)
}

\begin{abstract}
Riassunto: Ricerche condotte in alcuni anni da uno degli autori (G.P.) nel Parco Regionale del Corno alle Scale (Appennino Tosco-Emiliano) hanno permesso il rinvenimento di 10 specie mai segnalate nell'area protetta che, aggiunte ai dati della letteratura, costituiscono un popolamento di 30 specie tra Mantodea e Orthoptera. Di particolare rilievo risulta Bohemanella frigida, prima segnalazione per l'intera catena appenninica e Omocestus viridulus in quanto specie di notevole rarità. Il popolamento è costituito da specie angariane settentrionali e meridionali; da neoendemiti evoluti da elementi immigrati in Appennino durante le glaciazioni pleistoceniche; da specie immigrate in Appennino nel Quaternario. Tra le specie che caratterizzano l'intero popolamento emergono quattro endemiti appenninici: Pholidoptera aptera goidanichi, Stenobothrus apenninus, Podisma emiliae, Metrioptera caprai galvagnii.
\end{abstract}

\begin{abstract}
Orthoptera and Mantodea of the Parco Regionale del Corno alle Scale (Appennino Tosco-Emiliano): literature data and new records of boreo-orophylous elements (Insecta).

Researches ran by one of the authors (G.P.) in Parco Regionale del Corno alle Scale (Appennino Tosco-Emiliano) resulted in 10 species new for this protected area that, plus literature data, rise to 30 the species of Mantodea and Orthoptera known for this Park. Most interesting is the population of Bohemanella frigida, first finding for the appenine chain, and Omocestus viridulus, being this species very rare in the Appennini. The population is composed by southern and northern angarian species, by neoendemics evolved from elements immigrated in the Appennini during pleistocenic glaciations, and by species immigrated in the Appennini during the Quaternary. Within all the species found here, 4 appennine endemic species are more interesting: Pholidoptera aptera goidanichi, Stenobothrus apenninus, Podisma emiliae, Metrioptera caprai galvagnii.
\end{abstract}

Key words: Mantodea, Orthoptera, Appennino Tosco-Emiliano, population, biogeography, endemic species.

\section{INTRODUZIONE}

Il Parco Regionale del Corno alle Scale è situato nell'alto Appennino Bolognese, settore dell'Appennino Tosco-Emiliano; è caratterizzato da una copertura boschiva pressoché costante se si eccettua la parte cacuminale oltre 1600-1700 m di quota. La zona oltre il limite degli alberi è caratterizzata da brughiere e pascoli, con la presenza di ambienti rupestri relativi ai crinali e alle cime più elevate come lo stesso Corno alle Scale (1945 m), il Monte Spigolino (1827 m), il Monte Cupolino (1852 m) e il Monte La Nuda (1827 m).

Il Parco Regionale del Corno alle Scale confina a ovest con il Parco Regionale dell'Alto Appennino Modenese (o Parco del Frignano) (Fig. 1). L'area protetta è particolarmente interessante per gli aspetti faunistici, botanici, morfologici, climatici; questi ultimi hanno connotati di una certa variabilità, soprattutto nella stagione estiva. Sostanzialmente il clima è subatlantico, con aspetti microclimatici da individuare in alcune specifiche stazioni come lungo la Valle del Silla o la Valle del Dardagna, o come nei pressi dei crinali intorno a 1800-1900 m, dove si registrano temperature invernali molto rigide e temperature estive di frequente particolarmente contenute.

Questo studio presenta una visione d'insieme sulla fauna a Mantodea e Orthoptera di quest'area nord-appenninica relativamente alle ricerche effettuate in modo continuativo tra il 2005 e il 2010 e ai dati della letteratura, con le inedite segnalazioni della importante presenza ecologico-biogeografica di Bohemanella frigida (Boheman, 1846) ed Omocestus viridulus (Linnaeus, 1758).

Nell'Appennino Tosco-Emiliano, relativamente all'area indagata, le conoscenze fino ad ora acquisite sulle faune ortotterologiche scaturiscono dai dati della letteratura, presenti nelle opere di La Greca (1951; 1965), Baccetti (1952; 1954; 1957; 1958; 1963), Galvagni (1959; 1971), Pedroni (1997), Fontana (2003), Fontana et al. (2005a), Fontana et al. (2005b), Massa et al. (2012). 

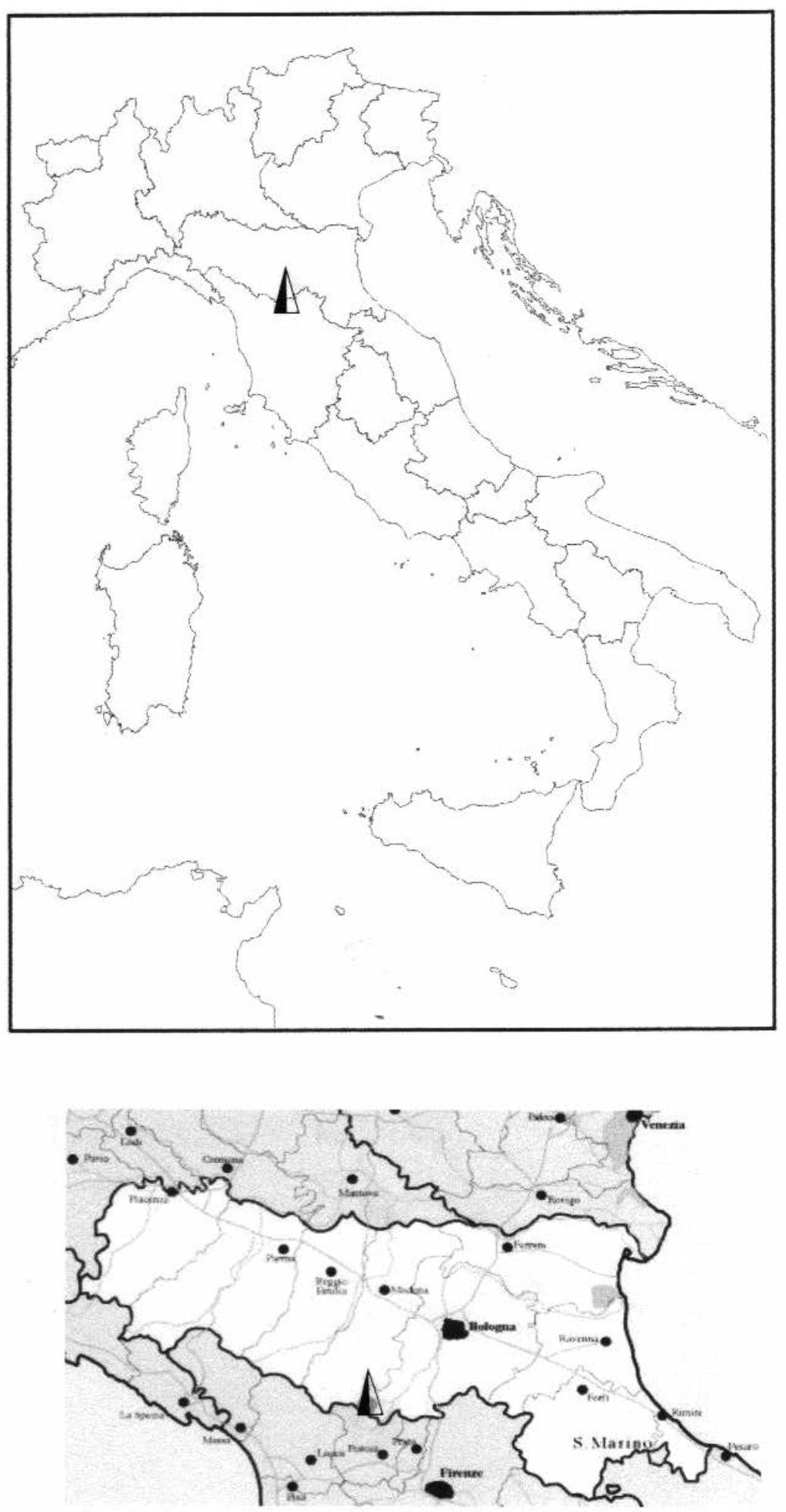

Fig. 1. Carta del Parco Regionale del Corno alle Scale (PCS). 


\section{Materiali e Metodi}

I metodi di raccolta sono quelli consueti per gli Ortotteri, quali: il retino da sfalcio; la raccolta a vista con barattoli in vetro di opportune dimensioni intrappolando gli insetti direttamente sulle diverse parti delle essenze erbacee; l'ombrello entomologico. Non sono state sistemate trappole a caduta, né sono state effettuate cacce notturne.

Tutte le specie sono state determinate (o confermate) dal secondo autore (F.M.B.) prendendo come riferimento i lavori di Harz (1969; 1975), Harz \& Kaltenbach (1976), Massa et al. (2012); le raccolte sono state effettuate dal primo autore (G.P.) così come la ricerca bibliografica; quest'ultima ha prodotto la segnalazione di 20 specie tra Mantodea e Orthoptera, alle quali vanno aggiunte altre 10 nuove segnalazioni per l'area indagata di cui una nuova per l'intera catena appenninica. Per le collezioni alle quali fa riferimento la letteratura si vedano i lavori recenti di Fontana et al. (2005a), Fontana et al. (2005b). Note di tipo ecologico sono state ricavate da Fontana et al. (2002) e Massa et al. (2012) le osservazioni dirette in ambiente vengono indicate caso per caso.
Tutti i reperti recenti sono nella collezione del primo autore (G.P.) (PED). Il lavoro considera anche le zone strettamente limitrofe al parco regionale, ciò significa che sono state indagate stazioni non lontane più di 2-3 chilometri dal confine dell'area protetta e comunque di ecosistemi senza soluzione di continuità oltre il confine del parco, perché senza alcuna variazione ecologica significativa. Le specificazioni "boreo-orofilo" e "boreo-alpino", che qui vengono seguite relativamente ad alcune specie, fanno riferimento alle indicazioni di Focarile (1974) che differenzia e definisce chiaramente l'uso di un termine rispetto all'altro.

I dati bibliografici si riferiscono alla zona protetta e alle stazioni strettamente limitrofe. Nei "reperti recenti" il Parco del Corno alle Scale ha come acronimo PCS.

\section{Elenco faunistico e Note ecologiche}

\section{MANTODEA}

1. Mantis religiosa religiosa Linnaeus, 1758 (Fig. 2) RePerTi ReCENTI. Monti della Riva (Valle del Dardagna), 800 m, VII.2007 (3 es.) Pedroni G. leg. (PED). Corologia e nOte ECologiche. Corotipo Ovest-Paleartico (WPA).

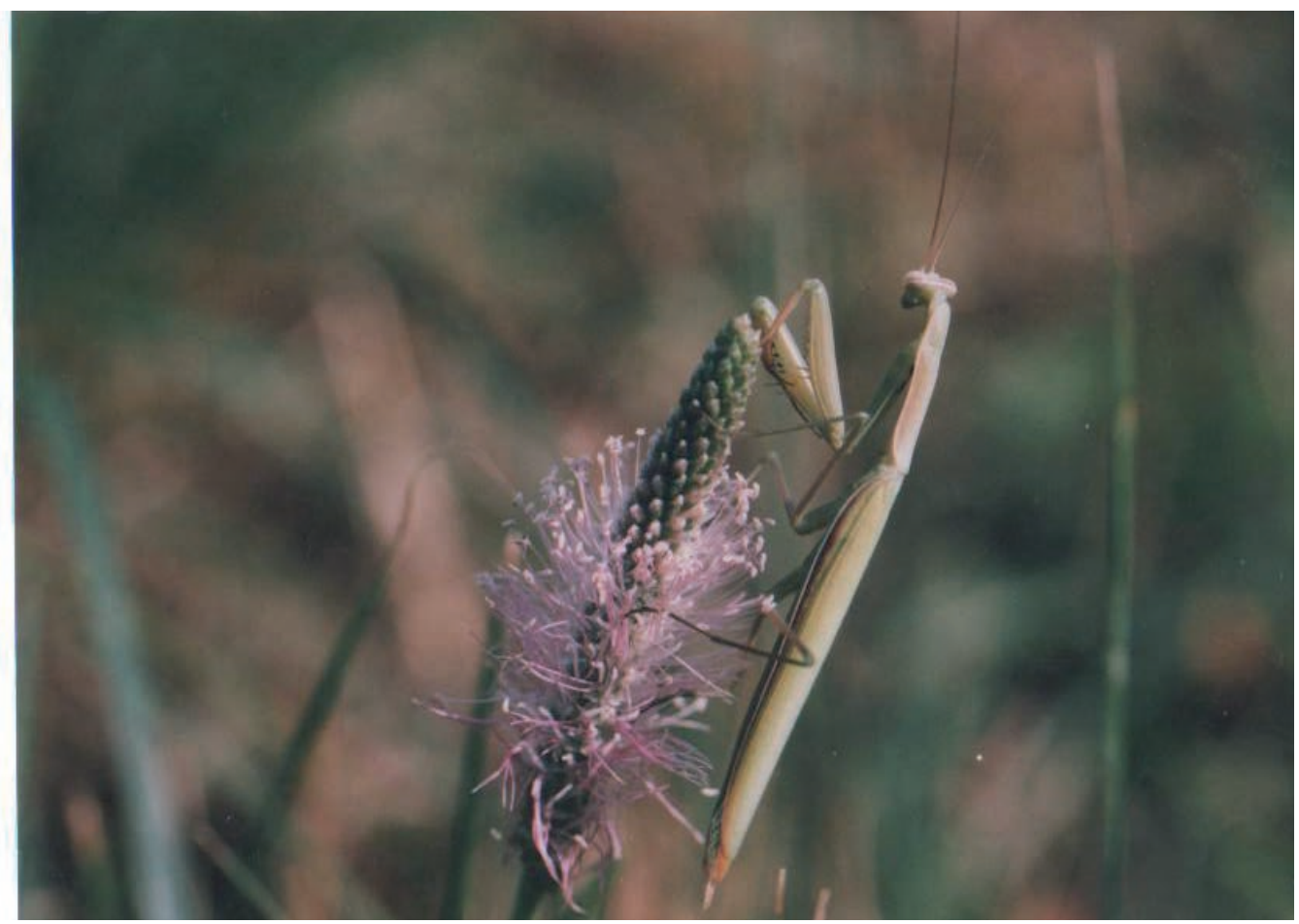

Fig. 2. Mantis religiosa religiosa, Monti della Riva, Valle del Dardagna (foto G.P. 2007). 
Dalle osservazioni degli ultimi tre anni risulta essere un ambiente idoneo ad ospitare una popolazione stabile di Mantis religiosa religiosa, come hanno dimostrato le perlustrazioni durante la stagione estiva, evidenziando sempre la presenza di ooteche e di adulti sulle essenze erbacee (osservazioni dirette in ambiente G.P.). Il versante verso il Torrente Dardagna (sud, sud-est) dei Monti della Riva è un ecosistema termofilo, con versanti caratterizzati da una notevole pendenza, di frequente quasi verticale. Su questi versanti, dove lo strato di suolo è molto spesso interrotto dagli affioramenti di arenaria macigno, è presente una copertura boschiva rada e discontinua; qui è presente in maggioranza il carpino nero (Ostrya carpinifolia).

\section{ORTHOPTERA}

\section{Meconema meridionale A. Costa, 1860}

DATI BIBLIOGRAFICI. Lago Scaffaiolo (Baccetti, 1952). Corologia e note ECOlogiche. Corotipo Europeo (EUR).

É una specie che vive su alberi e arbusti, ove è attiva di notte alla ricerca di piccole prede, come altri Insetti. Si rifugia sulla pagina inferiore delle foglie sia se disturbata, sia di giorno per ripararsi da insolazioni particolarmente intense, o durante periodi piovosi.

\section{Tettigonia cantans (Fuessly, 1775) (Figg. 3, 4)}

REPERTI RECENTI. Briglia del Baggioledo, PCS, 18.VIII.2006, 1600 m (1 es.) Pedroni G. leg. (PED). Corologia e note ecologiche. Corotipo AsiaticoEuropeo (ASE).

La specie risulta presente dal piano montano a quello alpino, in ecosistemi prativi o in radure limitrofe a boschi, con essenze erbacee particolarmente sviluppate, come Urtica sp.pl. (Fontana, 2003). È stata osservata in stazioni del parco caratterizzate da basse temperature, dalla Briglia del Baggioledo fino al crinale tra il Passo dello Strofinatoio e il Monte Cupolino (da 1600 fino a $1850 \mathrm{~m}$ ) (osservazioni dirette in ambiente G.P.).

4. Decticus verrucivorus verrucivorus (Linnaeus, 1758) REPERTI RECENTI. Rifugio Cavone, PCS, 15.VIII.2006, 1400 m (6 es.) Pedroni G. leg. (PED). DATI BIBLIOGRAFICI. Lago Scaffaiolo (Baccetti, 1963); crinale Corno alle Scale-Monte La Nuda (Pedroni, 1997).

Corologia e note ecologiche. Corotipo AsiaticoEuropeo (ASE).

La specie è onnivora, nutrendosi sia di Insetti sia di vegetali. Vive in praterie montane e in stazioni submontane.

\section{Platycleis grisea grisea (Fabricius, 1781)}

REPERTI RECENTI. Case Lanzi (Farnè), Valle del Dardagna, 15.VIII.2006, 750 m (1 es. ) Pedroni G. leg. (PED).

DATI BIBLIOGRAFICI. Lago Scaffaiolo (Baccetti, 1954) Corologia e note ecologiche. Corotipo Europeo (EUR).

Specie ad ampia valenza ecologica.

6. Metrioptera caprai galvagnii (Baccetti, 1963) (Fig. 5)

REPERTI RECENTI. Rifugio Cavone, PCS, 15.VIII.2006, 1400 m (2 es.) Pedroni G. leg. (PED). Corologia E note ECologiche. Corotipo Sud-Europeo (SEU) specie boreo-orofila a distribuzione Appenninica (APP).

Il Parco del Corno alle Scale risulta la stazione più orientale dell'areale, che interessa l'Appennino Ligure e Tosco-Emiliano. La specie popola stazioni dal limite superiore della fascia montana fino alle stazioni cacuminali delle alte quote appenniniche, fino a 1800 $\mathrm{m}$ (Fontana, 2003); la sua dieta è condizionata alla presenza della graminacea Brachypodium pinnatum P.B. Presenza e diffusione di Metrioptera caprai galvagnii (e altre sottospecie conspecifiche) sono di tipo relittuale relativamente alla glaciazione Würmiana (Baccetti, 1963).

\section{Bicolorana bicolor bicolor (Philippi, 1830)}

Reperti recenti. Cà Berna, Valle del Dardagna, PCS (BO), 1000 m, 10.VIII.2006 (1 es.) Pedroni G. leg. (PED).

Corologia E note eCologiche. Corotipo Europeo (EUR).

Specie mesoigrofila stenoterma, popola zone praticole particolarmente umide e ricche di graminacee.

\section{Sepiana sepium (Yersin, 1854)}

DATI BIBLIOGRAFICI. Lago Scaffaiolo (Baccetti, 1954) Corologia e note ecologiche. Corotipo Centroasiatico-Mediterraneo (CAM).

É una specie chiaramente termofila o xerotermofila. Vive dal livello del mare fino a circa $1600 \mathrm{~m}$ in Appennino; al Lago Scaffaiolo è arrivata a $1800 \mathrm{~m}$ di quota. E' possibile che sia meno xerotermofila di quanto noto. 
Il popolamento a Orthoptera e Mantodea del Parco Regionale del Corno alle Scale

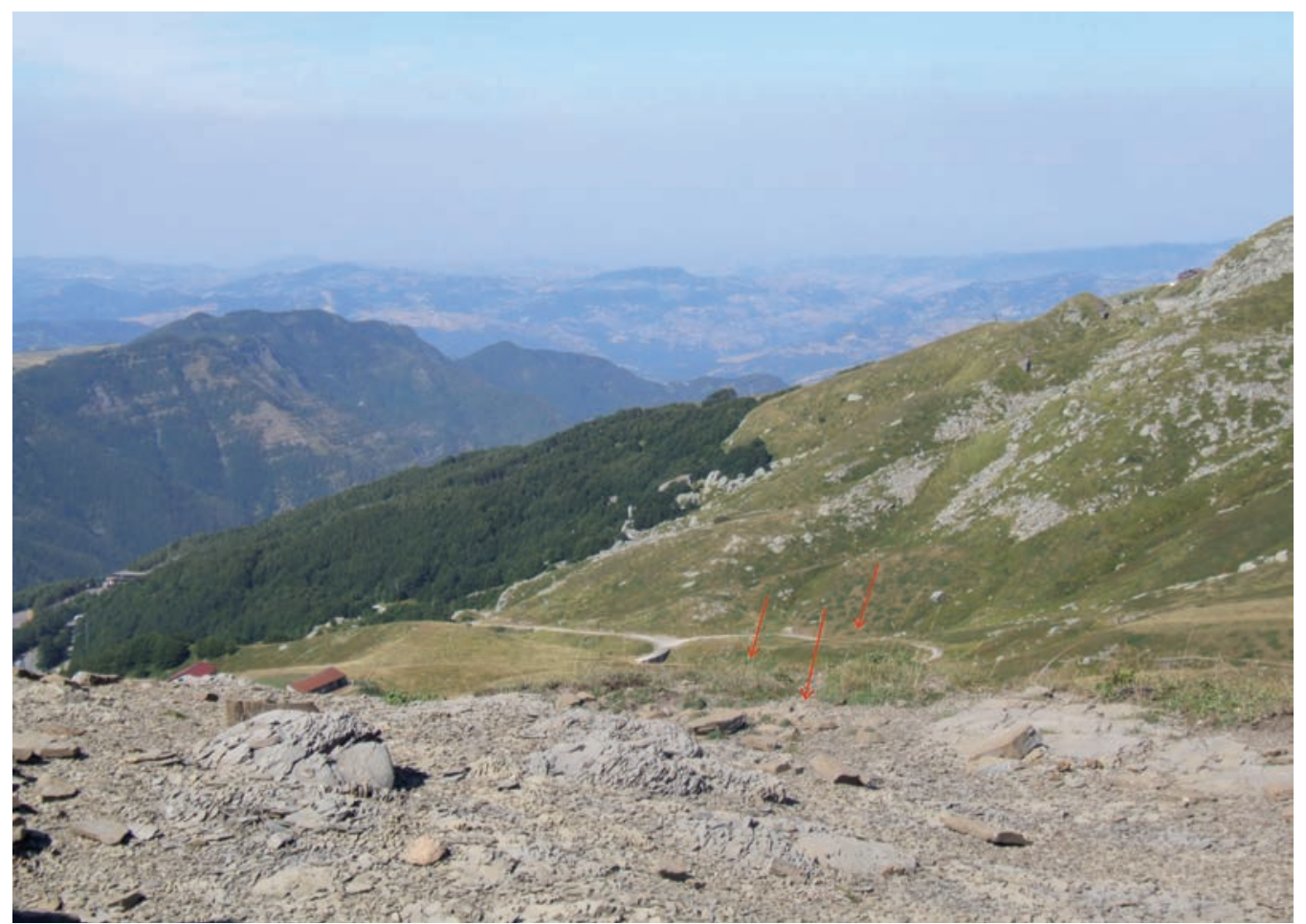

Fig. 3. Briglia del Baggioledo (PCS), stazione indicata dalle frecce rosse (foto G.P. 2008).

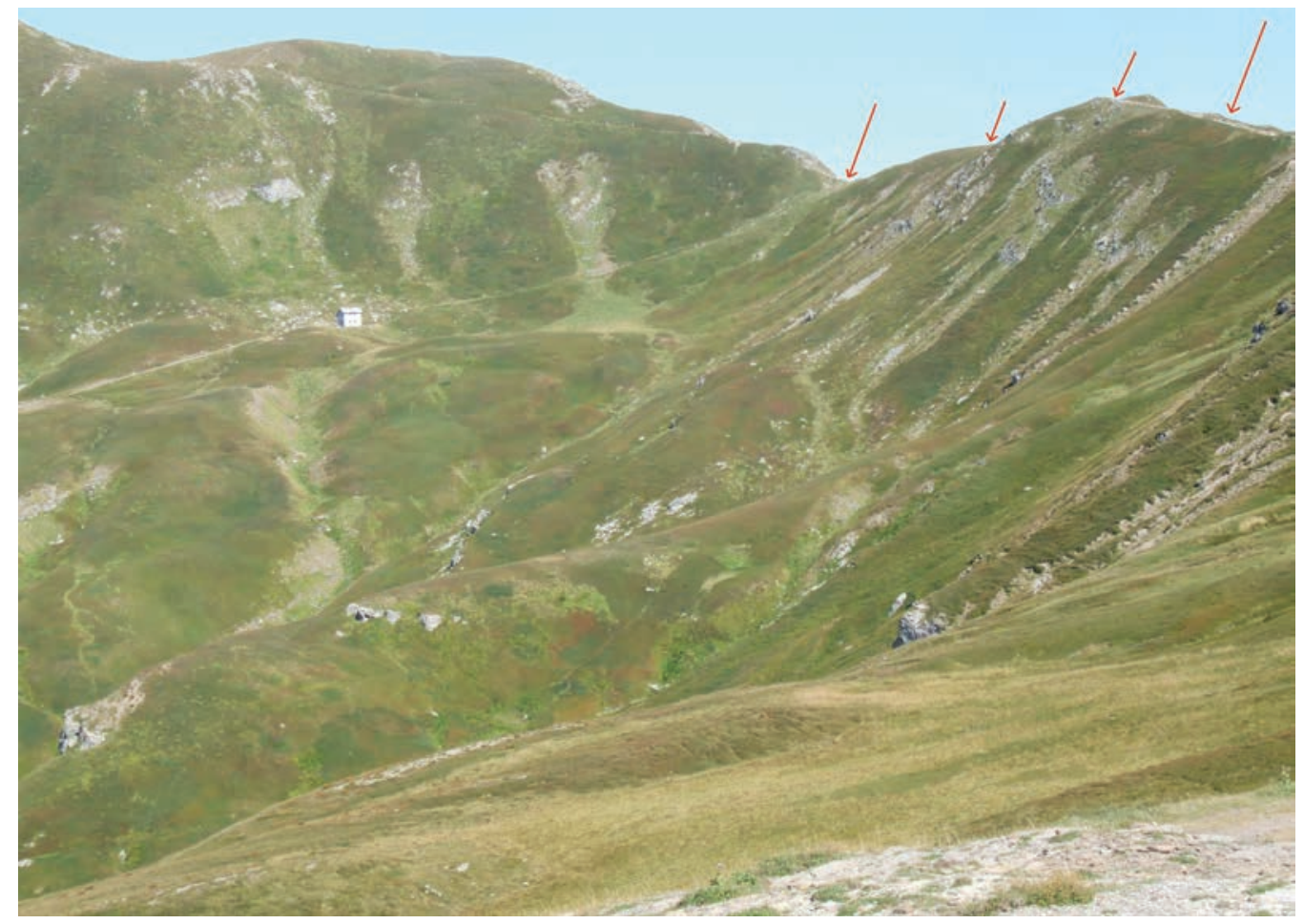

Fig. 4. Crinale dal Passo dello Strofinatoio verso il Monte Cupolino (PCS) (1800-1850 m) (foto G.P. 2008). 
9. Pholidoptera aptera goidanichi (Baccetti, 1963) (Figg. 3, 6)

Reperti ReCenti. Briglia del Baggioledo, PCS, 18.VIII.2006, $1600 \mathrm{~m}$ (1 es.) Pedroni G. leg. (PED). Rifugio Cavone, PCS, 23.VII.2010, 1400 m (1 es.) Pedroni G. leg.

Corologia e note eCologiche. Corotipo Sud-Europeo (SEU) specie boreo-orofila a distribuzione Appenninica (APP).

Elemento appenninico di stazioni dalla Liguria fino al Lazio, dove lo si rinviene da $900 \mathrm{~m}$ fino a $1900 \mathrm{~m}$. Vive in radure ai margini di boschi di latifoglie e su versanti anche ripidi (Fontana, 2003). La sottospecie è stata identificata sulla base dei caratteristici titillatori.

\section{Pholidoptera fallax (Fischer, 1854)}

Dati BiBLIOGRAFici. Lago Scaffaiolo (Baccetti, 1954) Corologia e note ecologiche. Corotipo TuranicoEuropeo (TUE).

Specie ad ampia valenza ecologica.

\section{Eupholidoptera chabrieri chabrieri (Charpentier,} 1825)

DATI BIBLIOGRAFICI. Lago Scaffaiolo (Baccetti, 1954) NotA. L'identificazione delle Eupholidoptera è piuttosto delicata, e la determinazione si basa quanto meno sul carattere morfologico dei titillatori maschili che consentono di ascrivere i reperti al taxon specifico.

Corologia e nOte ECOlogiche. Corotipo Ovest-Europeo (W-EUR).

Elemento orofilo, non comune, di praterie alto-montane, evoluta da una specie nord-egeica (La Greca, 1965).

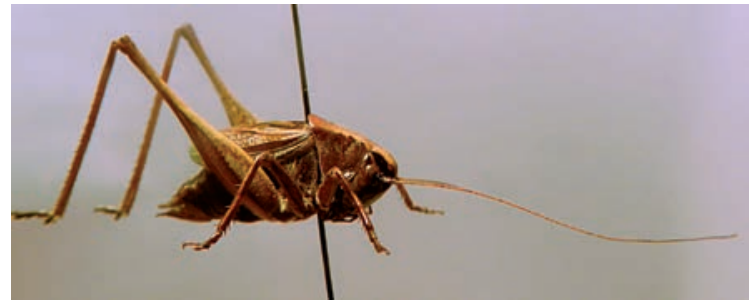

Fig. 5. Metrioptera caprai galvagnii, Rifugio Cavone (PCS) (foto M.K. Pedroni 2012).
12. Rhacocleis germanica (Herrich-Schaeffer, 1840) DATI BIBLIOGRAFICI. Lago Scaffaiolo (Baccetti, 1954) Corologia e note eCologiche. Corotipo Sud-Europeo (SEU).

La specie popola ecosistemi termofili dalla pianura alla montagna fino a circa $1200 \mathrm{~m}$; al lago Scaffaiolo si presenta ad un'altezza di $1800 \mathrm{~m}$ quindi oltre il suo normale limite di popolamento in quota; di norma vive in ecotoni con presenza di vegetazione arbustiva isolata. La sua dieta è a carattere zoofago.

13. Gryllus campestris Linnaeus, 1758

DATI BiBLIOGRAFici. Lago Scaffaiolo (Baccetti, 1954) Corologia e nOte ECOLOGiche. Corotipo Paleartico (PAL).

Specie ad ampia valenza ecologica; infatti gli esemplari della specie si rinvengono in ambienti praticoli dalla pianura fino alla montagna (1300-1400 m).

14. Nemobius sylvestris (Bosc, 1792)

RePERTI RECENTI. Farnè, Valle del Dardagna, $700 \mathrm{~m}$, 18.VIII.2006 (1 es.) Pedroni G. leg. (PED).

Corologia e note eCologiche. Corotipo EuropeoMediterraneo (EUM).

Specie saprofaga, geofila e mesofila presente in strati superficiali di suoli in boschi termofili. Il ciclo biologico della specie è legato alle lettiere dove si trovano anche gli esemplari adulti.

15. Psophus stridulus stridulus Linnaeus, 1758

Dati Bibliografici. Corno alle Scale (Baccetti, 1958); Lago Scaffaiolo (Baccetti, 1954).

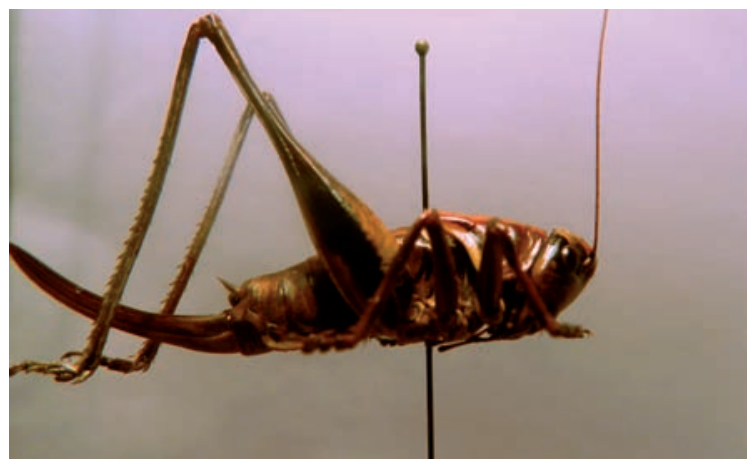

Fig. 6. Pholidoptera aptera goidanichi, Briglia del Baggioledo (PCS) (foto M.K. Pedroni 2012). 
Corologia e note ecologiche. Corotipo Paleartico (PAL).

Le due stazioni indicate dalla letteratura della zona indagata rimangono le più sud-orientali dell'areale italiano della specie.

\section{Podisma emiliae Ramme, 1926 (Fig. 7)}

Reperti Recenti. Rifugio Cavone, PCS, 1400 m, 15.VIII.2006 (4 es.) Pedroni G. leg. (PED).

Rifugio Cavone, PCS, 1400 m, 23.VII.2010 (2 es.) Pedroni M.K. leg. (PED).

Baita del Sole, PCS, 1500 m, 24.VII.2010 (5 es.) Pedroni G. leg. (1 es) Pedroni M.K. (PED).

Dati Biblografici. Corno alle Scale e Passo della Croce Arcana (MO-PT) (Baccetti, 1957); Lago Scaffaiolo (La Greca, 1951; Galvagni, 1971; Pedroni, 1997). Corologia E note ECOLOGICHE. Corotipo Sud-Europeo (SEU) specie boreo-orofila a distribuzione NordAppenninica (APP).

Endemita dell'Appennino Tosco-Emiliano, trova la sua origine a seguito dei processi glaciali würmiani. È specie delle praterie cacuminali o di suoli sassosi con rada vegetazione, fino a circa $1800 \mathrm{~m}$ (Fontana, 2003).

\section{Bohemanella frigida (Boheman, 1846) (Fig. 8)}

REPERTI RECENTI. Farnè, Valle del Dardagna, $650 \mathrm{~m}$, VIII.2007 (1 es.) Pedroni G. leg. (PED).

Corologia E note ECologiche. Corotipo Olartico (OLA).

Importante prima segnalazione per la catena appenninica di specie fino ad ora presente nella sola catena alpina; brachittera con tegmine non più lunghe di metà addome. Ottimo bioindicatore. Esemplare rac-

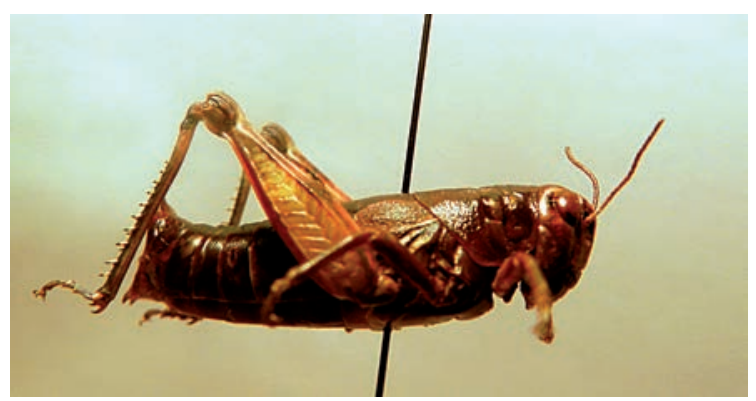

Fig. 7. Podisma emiliae, Rifugio Cavone (PCS) (foto M.K. Pedroni 2012). colto sulle erbe in zona ombrosa e umida, in zona ripariale lungo il torrente Dardagna (osservazioni dirette in ambiente G.P.).

La specie eualpina, microterma, è presente in stazioni cacuminali tra 2000 e 2900 m nell'arco alpino, su versanti freddi, scarsamente soleggiati con vegetazione bassa o rada. La presenza di questa specie in stazione nord-appenninica può essere interpretata invocando una sua relittualità post-würmiana; la cattura dell'esemplare di Farnè potrebbe essere occasionale relativamente alla quota; si dovranno comunque attendere ulteriori catture per confermare la sua presenza in Appennino, che porterebbero a delinearne un areale disgiunto relativamente alla penisola italiana. Nota Sistematica Skareas \& Hsiung (1999) hanno messo in evidenza che la sola specie boreo-orofila presente in Europa ed anche in Italia, determinata fino a poco tempo fa sub Melanoplus frigidus frigidus, sistematicamente deve essere inserita nella tribù dei Podismini e non in quella dei Melanoplini; Chapco \& Litzenberger (2002), pur non condividendo questa opinione, considerano Melanoplus polifiletico. Il volume sugli Orthoptera della Fauna d'Italia (Massa et al., 2012) riporta la specie sub Bohemanella frigida.

\section{Anacridium aegyptium (Linnaeus, 1764)}

REPERTI RECENTI. Farnè, Valle del Dardagna, 700 m, VII.2005 (1 es.) Pedroni G. leg. (PED).

DATI BIBLIOGRAFICI. Lago Scaffaiolo (Baccetti, 1952) Corologia E nOte ECOLOGICHE. Corotipo Afrotropicale-Mediterraneo (AFM).

Specie capace di una buona adattabilità a diversi tipi di ambienti, dalla pianura alla montagna, e di clima, con una notevole capacità migratoria.

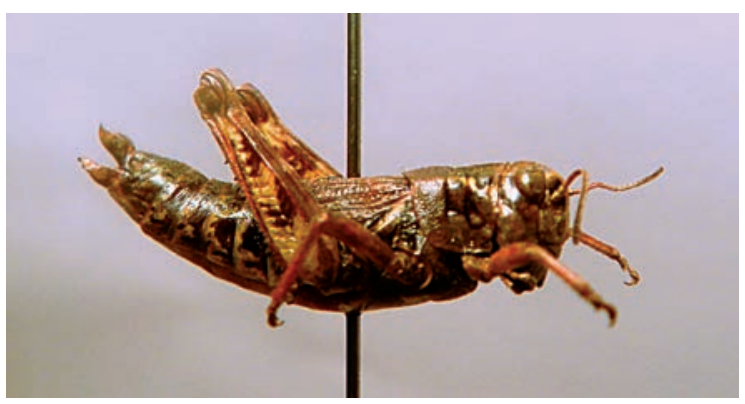

Fig. 8. Bohemanella frigida, Farnè, Valle del Dardagna (foto M.K. Pedroni 2012). 
19. Oedipoda germanica (Latreille, 1804)

REPERTI RECENTI. Farnè, Valle del Dardagna, $700 \mathrm{~m}$, VII.2006 (1 es.) Pedroni G. leg. (PED).

Rifugio Cavone, PCS, 1400 m, 15.VIII.2006 (1 es.)

Pedroni G. leg. (PED).

Corologia e note ecologiche. Corotipo TuranicoEuropeo (TUE).

Specie dalle spiccate tendenze termofile. Esemplare di Farnè raccolto in radura brulla e soleggiata.

20. Omocestus rufipes (Zetterstedt, 1821)

Dati Bibliografici Lago Scaffaiolo (Baccetti, 1954) Corologia e note eCologiche Corotipo Paleartico (PAL).

Specie ad ampia valenza ecologica, mesoxerofila, più raramente mesofila. Nella stazione del parco dove è stata raccolta $(1800 \mathrm{~m})$ si comporta, appunto, come specie mesoigrofila.

\section{Omocestus viridulus (Linnaeus, 1758) (Fig. 9)}

RePERTI RECENTI. Rifugio Cavone, PCS, 15.VIII.2006, $1400 \mathrm{~m}$ (1 es.) Pedroni G. leg. (PED). Corologia e note ecologiche. Corotipo SibiricoEuropeo (SIE).

La specie risulta rarissima in Appennino, la zona del Cavone è solo la terza segnalazione per l'intera catena appenninica. Popola ambienti di alta quota nelle Alpi da 1500 a $2000 \mathrm{~m}$ circa, più di rado al di sotto di 1000 $\mathrm{m}$, in ecosistemi prativi, di torbiera, di palude, ma senza particolari esigenze di umidità. Molto spesso vive in sintopia con Aeropus s. sibiricus (presente nel parco) e Podisma pedestris.

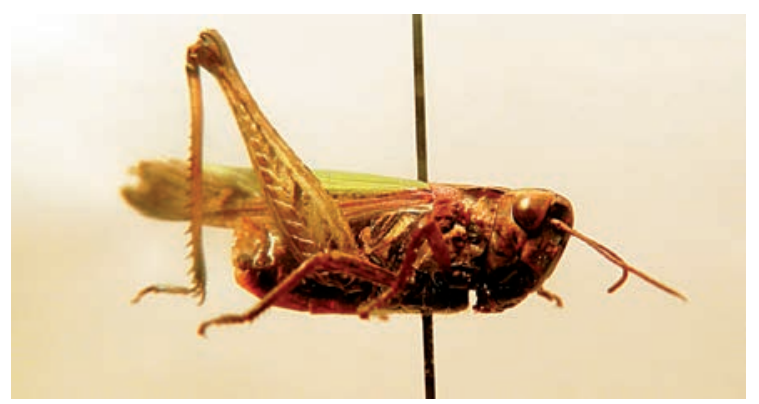

Fig. 9. Omocestus viridulus, Rifugio Cavone (PCS) (foto M.K. Pedroni 2012).
22. Stenobothrus apenninus Ebner, 1915

Dati Biblografici. Lago Scaffaiolo, Sestola (La Greca, 1951; Galvagni, 1959; Pedroni, 1997); Corno alle Scale (Galvagni, 1971)

Corologia e note ECOLOGiche. Corotipo Sud-Europeo (SEU) specie boreo-orofila a distribuzione Appenninica (APP).

Specie endemica appenninica, orofila, fino alle quote più elevate della catena appenninica. Stenobothrus apenninus potrebbe essere un elemento immigrato in Appennino soprattutto nel Quaternario per porte di accesso alpino-occidentali nel corso di periodi glaciali caratterizzati da oscillazioni climatiche fredde (La Greca, 1965).

\section{Stenobothrus lineatus lineatus (Panzer, 1796)}

DATI BIBLIOGRAFICI. Lago Scaffaiolo (Baccetti, 1954) Corologia e note ecologiche. Corotipo AsiaticoEuropeo (ASE).

Specie con buona capacità di adattamento a vari tipi di ecosistemi, mesoxerofila e più di rado mesoigrofila Vive in ambienti montani fino a circa $2500 \mathrm{~m}$ su Alpi e Appennini; il ciclo biologico è legato alla presenza di graminacee alla base delle quali, subito sotto la superficie del terreno, vengono deposte le ooteche (Fontana et al., 2002).

\section{Aeropus sibiricus sibiricus (Linnaeus, 1767)}

(Figg. 10, 11)

Dati Bibliografici. Corno alle Scale, circo glaciale del Cavone, 1800 m, 9.VII.1995 (1 es.) Pedroni G. leg. (Pedroni, 1997 sub Gomphocerus sibiricus) (PED).

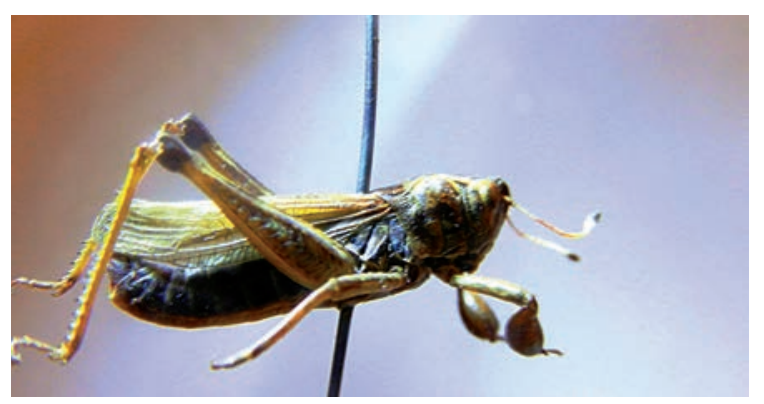

Fig. 10. Aeropus sibiricus sibiricus, Corno alle Scale verso la cima (1900 m) (Foto M.K. Pedroni 2012). 
Corologia E note ecologiche. Corotipo AsiaticoEuropeo (ASE).

Specie eurosibirica eualpina, che si rinviene di norma alle alte quote su Alpi e Appennini. Nella catena appenninica la sua distribuzione è discontinua, dove la specie è da considerarsi un tipico relitto glaciale würmiano (La Greca, 1951). Adulti della specie sono stati osservati fino a 3000 m nella Grande Sassière (Francia) (Voisin, 1995). Al Colle de l'Iseran (Francia) sono stati osservati numerosi esemplari adulti della specie fino a $3150 \mathrm{~m}$ di quota e oltre in ambienti sassosi e particolarmente spogli di vegetazione, anche con tempo nuvoloso, temperature prossime a $0^{\circ} \mathrm{C}$ e con vento forte (Pedroni, 1998). In Appennino settentrionale è presente in ecosistemi oltre il limite della vegetazione arborea e arbustiva, con predominanza di vegetazione erbacea radente il suolo.

\section{Gomphocerus rufus (Linnaeus, 1758)}

REPERTI RECENTI. Cà Berna, Valle del Dardagna, PCS (BO), 1000 m, 10.VIII.2006 (1 es.) Pedroni G. leg. (PED).

Dati BIBliografici. Lago Scaffaiolo (Baccetti, 1954). Corologia e note ecologiche. Corotipo AsiaticoEuropeo (ASE).

Specie con caratteri mesotermofili, più raramente colonizza ambienti con diversi gradi di umidità, prediligendo zone con buona luminosità; popola, infatti, margini dei boschi, radure, sentieri in boschi luminosi e prati con vegetazione arbustiva rada dal piano

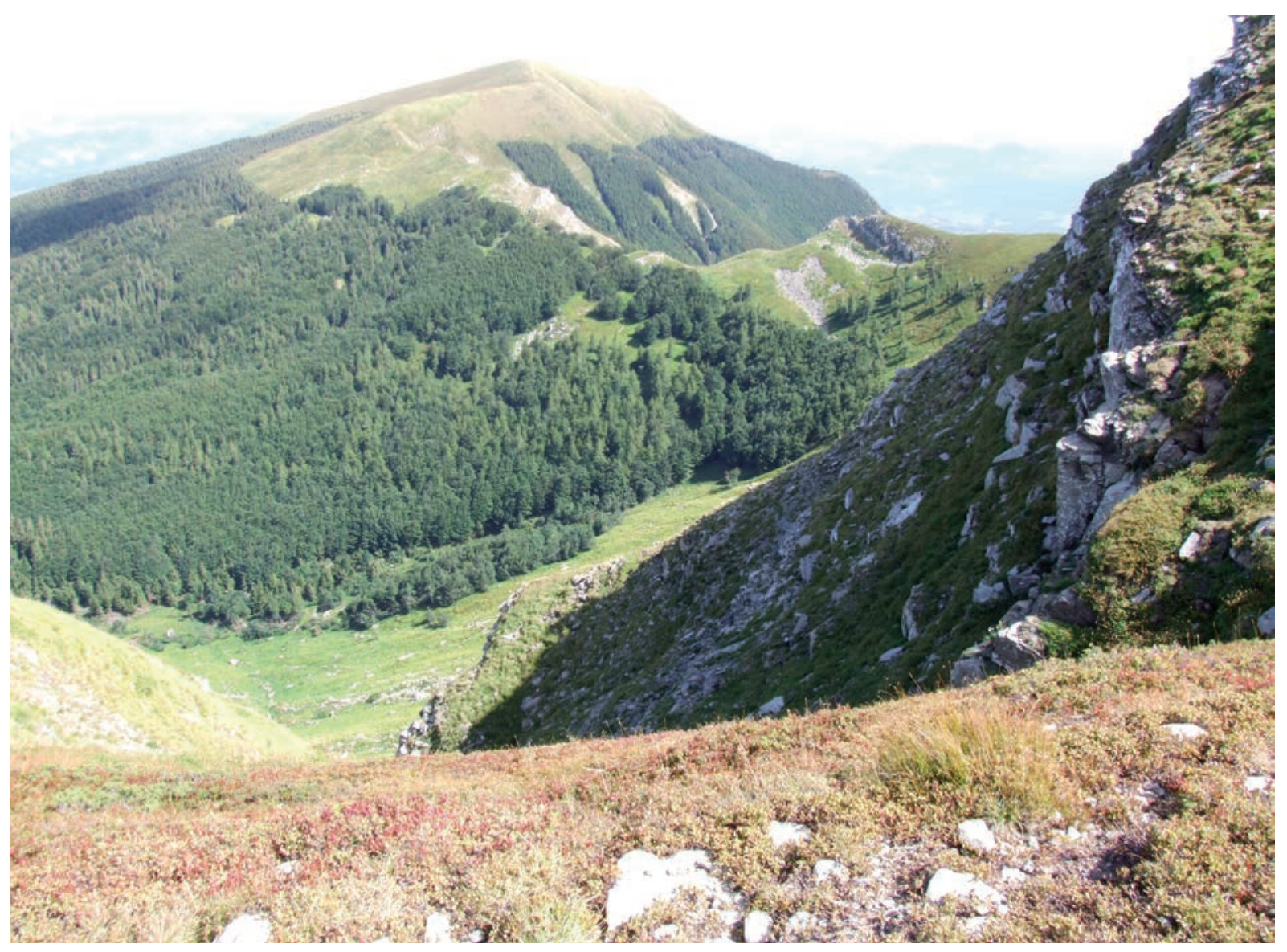

Fig. 11. Corno alle Scale (1945 m) (foto G.P. 2008). 
subalpino fino a circa $2400 \mathrm{~m}$ su Alpi e Appennini. Le ooteche vengono deposte in piccole cavità del suolo chiudendone l'apertura raspando con le zampe posteriori il terreno (Fontana et al., 2002).

26. Stauroderus scalaris scalaris (Fischer de Waldheim, 1846)

DATI BiBLIOGRAFICI. Lago Scaffaiolo (Baccetti, 1954) Corologia e nOte ECologiche. Corotipo Sud-Europeo (SEU).

Specie montana di norma legata ad ecosistemi prativi, diffusa alle quote medio-alte in tutta l'Italia peninsulare.

27. Chorthippus dorsatus dorsatus (Zetterstedt, 1821) Dati BIBLIOGRAFICI. Lago Scaffaiolo (Baccetti, 1954) Corologia e note ecologiche. Corotipo SibiricoEuropeo (SIE).

Specie mesoigrofila, colonizza vari tipi di ambienti, dai connotati fresco-umidi, dalla pianura fino a circa $1800 \mathrm{~m}$ di quota su Alpi e Appennini, ma con buona naturalità.

28. Chorthippus parallelus parallelus (Zetterstedt, 1821)

RePerTi ReCENTI. Cà Berna, Valle del Dardagna, PCS, 1000 m, 10.VIII.2006 (2 es.) Pedroni G. leg. (PED). Rifugio Cavone, PCS, 15.VIII.2006, 1400 m (5 es.) Pedroni G. leg. (PED).

Baita del Sole, Valle del Dardagna, PCS, $1500 \mathrm{~m}$, 24.VII.2010 (3 es.) Pedroni G. leg. (PED).

DATI BIBLIOGRAFicI. Lago Scaffaiolo (Baccetti, 1954) Corologia e note ecologiche. Corotipo SibiricoEuropeo (SIE).

Specie ad ampia valenza ecologica; a seconda del tipo di ecosistema popolato si può comportare come specie mesoigrofila o chiaramente igrofila, raggiungendo anche $2500 \mathrm{~m}$ di altezza su Alpi e Appennini.

29. Glyptobothrus brunneus brunneus (Thunberg, 1815)

DATI BIBLIOGRAFICI. Lago Scaffaiolo (Baccetti, 1954) Corologia e note ecologiche. Corotipo SibiricoEuropeo (SIE).

Specie ad ampia valenza ecologica, divenendo orofila man mano che si procede verso sud nella nostra penisola.

30. Euchorthippus declivus (Brisout, 1848)

REPERTI RECENTI. Farnè, Valle del Dardagna, $700 \mathrm{~m}$ 24.VII.2010 (9 es.) Pedroni G. leg. (PED).
Case Lanzi (Farnè), Valle del Dardagna, $650 \mathrm{~m}$, 24.VII.2010 (1 es.) Pedroni G. leg. (PED).

Corologia E nOte ECOLOGiche. Corotipo Europeo (EUR).

É una specie con tendenza xerofila, abita prati secchi, assolati e sassosi; a volte si comporta come elemento pseudo-igrofilo, ai margini di boschi o arrivando anche oltre il limite degli alberi.

\section{DisCUSSIONE}

Allo stato attuale delle conoscenze il popolamento a Mantodea e Orthoptera del Parco Regionale del Corno alle Scale (Appennino Tosco-Emiliano) (Figg. 1, 12) è caratterizzato da 30 specie, pari al $28,5 \%$ del popolamento globale dell'Appennino Tosco-Emiliano-Romagnolo, caratterizzato da poco più di 100 specie (Fontana et al., 2005a; Fontana et al., 2005b; Massa et al., 2012).

In alcuni anni di ricerca si è potuto riunire, attraverso raccolte dirette di uno degli autori (G.P.) e il reperimento dei dati in letteratura, una mole significativa di informazioni che ha permesso di evidenziare alcune caratteristiche biogeografiche, ecologiche e geonemiche di questo popolamento, ponendolo tra quelli più significativi dell'Appennino settentrionale. In un recente lavoro sugli Artropodi dell'orizzonte del faggio nell'Appennino settentrionale (Cerretti et al., 2003) si portava a conoscenza un popolamento ortotterologico di cinque riserve nord-appenniniche caratterizzato da 18 specie per 14 generi (Fontana, 2003); a questi dati va aggiunta un'altra specie, riportata nel lavoro di Bardiani \& Buzzetti (2009), che porta quel popolamento ad un totale di 19 specie. Il popolamento del Corno alle Scale, alla luce anche di queste ultime osservazioni, risulta pertanto di un rilevante interesse qualitativo. Nella Tab. 1 vengono riunite tutte le specie con alcune utili informazioni. L'analisi delle stazioni dove sono stati rinvenuti i reperti mette in evidenza che praticamente tutti gli esemplari sono stati catturati in ambienti aperti di radure tra boschi o ai margini di essi oppure in praterie cacuminali.

Prendendo in esame la distribuzione delle 30 specie (per un totale di 26 generi) sinora segnalate nell'area indagata, sulla base dei corotipi biogeografici fondamentali di riferimento (Stoch \& Vigna Taglianti, $2005)$, vengono proposti i raggruppamenti riportati in Tab. 1. In particolare emergono due presenze molto importanti entrambe inserite nel gruppo A:

- in primo luogo la presenza di Bohemanella frigida; 
è elemento che finora si considerava avere una distribuzione prettamente di tipo alpino. E' sicuramente una segnalazione di grande valore ecologico e biogeografico in quanto i dati qui esposti rappresentano la prima stazione per la catena appenninica. Troviamo questa specie nella Valle del Dardagna, valle con caratteristiche microclimatiche frescoumide per la maggior parte dell'anno, in particolare lungo il corso del torrente dove è presente una vegetazione ripariale e fitte foreste con elementi relitti würmiani, come le specie vegetali di sottobosco del genere Pirola sp.pl.;

- in secondo luogo la presenza di Omocestus viridulus; la specie risulta molto significativa in quanto elemento rarissimo in Appennino. Presenta un co- rotipo Sibirico-Europeo; si rinviene di norma nella catena alpina; nell'Appennino Tosco-Romagnolo e Marchigiano la si rinviene solo in 2 stazioni. Il Parco del Corno alle Scale rappresenta la prima segnalazione per l'Appennino Tosco-Emiliano.

Altro elemento di pregio ecologico risulta la specie boreo-orofila Aeropus sibiricus sibiricus, in quanto la sua presenza è da far risalire a dinamiche di popolamento imputabili alla glaciazione Würmiana, così come la sua presenza nell'Appennino centrale; il Corno alle Scale è l'unica stazione nord-appenninica fino ad ora accertata per questa specie.

Le quattro specie del gruppo $\mathrm{C}$ sono particolarmente significative in quanto elementi endemici, orofili, con areale nord-appenninico,

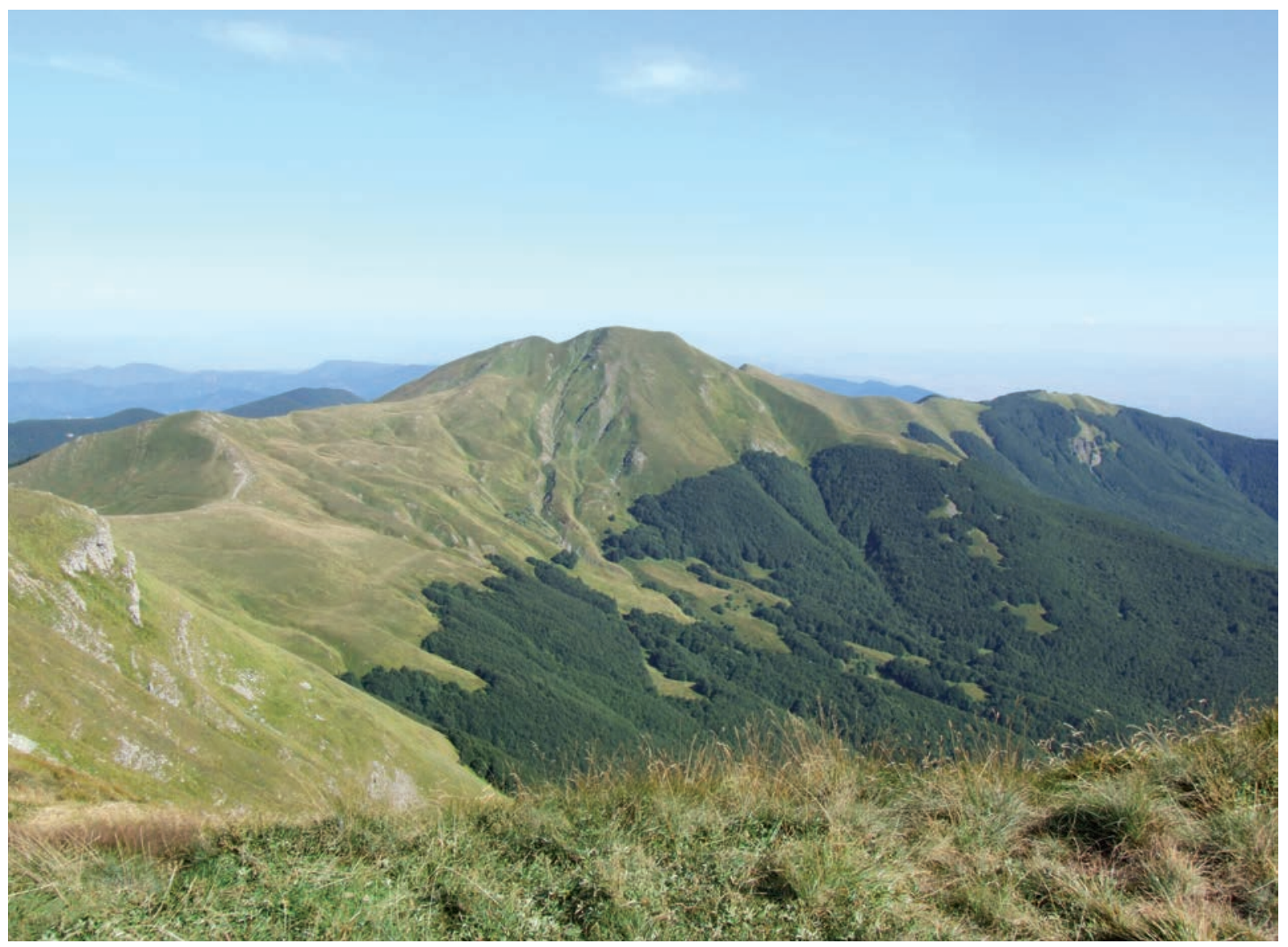

Fig. 12. Monte Gennaio con il crinale che si sviluppa dalle propaggini del Corno alle Scale (foto G.P. 2008). 
Tab. 1. Riepilogo tassonomico del popolamento ortotterologico del Parco Regionale del Corno alle Scale (Appennino ToscoEmiliano) con ripartizione delle specie nei quattro gruppi biogeografici e secondo i relativi corotipi.

$\mathbf{E}=$ specie endemica dell'Appennino settentrionale; $\mathbf{N S}=$ nuova segnalazione per la catena appenninica; $\mathbf{N}=$ specie mai segnalata nelle stazioni del parco; $\mathbf{N R}=$ nuovi reperti di specie già segnalate in letteratura; $\mathbf{Q}=$ quota massima raggiunta dalla specie nella zona indagata; $\mathbf{R}=$ specie rara in Appennino settentrionale.

\section{GRUPPO A}

17. Bohemanella frigida (Boheman, 1846)

13. Gryllus campestris Linnaeus, 1758

15. Psophus stridulus stridulus Linnaeus, 1758

20. Omocestus rufipes (Zetterstedt, 1821)

1. Mantis religiosa religiosa Linnaeus, 1758

3. Tettigonia cantans (Fuessly, 1775)

4. Decticus verrucivorus verrucivorus (Linnaeus, 1758)

23. Stenobothrus lineatus (Panzer, 1796)

24. Aeropus sibiricus sibiricus (Linnaeus, 1767)

25. Gomphocerus rufus (Linnaeus, 1758)

10. Pholidoptera fallax (Fischer, 1854)

19. Oedipoda germanica (Latreille, 1804)

21. Omocestus viridulus (Linnaeus, 1758)

27. Chorthippus dorsatus dorsatus (Zetterstedt, 1821)

28. Chorthippus parallelus parallelus (Zetterstedt, 1821)

29. Glyptobothrus brunneus brunneus (Thunberg, 1815)

8. Sepiana sepium (Yersin, 1854)
14. Nemobius sylvestris (Bosc, 1792)

\begin{tabular}{|c|c|c|c|c|}
\hline OLA & NS & $\mathrm{N}$ & & $\mathrm{Q}=700 \mathrm{~m}$ \\
\hline PAL & & & & $\mathrm{Q}=1800 \mathrm{~m}$ \\
\hline PAL & & & & $\mathrm{Q}=1900 \mathrm{~m}$ \\
\hline PAL & & & & $\mathrm{Q}=1800 \mathrm{~m}$ \\
\hline W-PAL & & $\mathrm{N}$ & & $\mathrm{Q}=800 \mathrm{~m}$ \\
\hline ASE & & $\mathrm{N}$ & & $\mathrm{Q}=1850 \mathrm{~m}$ \\
\hline ASE & & & NR & $\mathrm{Q}=1800 \mathrm{~m}$ \\
\hline ASE & & & & $\mathrm{Q}=1800 \mathrm{~m}$ \\
\hline ASE & & & & $\mathrm{Q}=1900 \mathrm{~m}$ \\
\hline ASE & & & NR & $\mathrm{Q}=1800 \mathrm{~m}$ \\
\hline TUE & & & & $\mathrm{Q}=1800 \mathrm{~m}$ \\
\hline TUE & & $\mathrm{N}$ & & $\mathrm{Q}=1400 \mathrm{~m}$ \\
\hline SIE & & $\mathrm{N}$ & & $\mathrm{Q}=1400 \mathrm{~m}$ \\
\hline SIE & & & & $\mathrm{Q}=1800 \mathrm{~m}$ \\
\hline SIE & & & NR & $\mathrm{Q}=1800 \mathrm{~m}$ \\
\hline SIE & & & & $\mathrm{Q}=1800 \mathrm{~m}$ \\
\hline EUM & & & & $\mathrm{Q}=700 \mathrm{~m}$ \\
\hline CAM & & & & $\mathrm{Q}=1800 \mathrm{~m}$ \\
\hline
\end{tabular}

\section{GRUPPO B}

2. Meconema meridionale A. Costa, 1860

EUR

5. Platycleis grisea grisea (Fabricius, 1781)

EUR

EUR

7. Bicolorana bicolor bicolor (Philippi, 1830)

EUR

30. Euchortippus declivus (Brisout, 1848)

W-EUR

11. Eupholidoptera chabrieri chabrieri (Charpentier, 1825)

SEU

12. Rhacocleis germanica (Herrich-Schaeffer, 1840)

26. Stauroderus scalaris scalaris (Fischer de Waldheim, 1846)
$\mathrm{Q}=1800 \mathrm{~m}$

$\mathrm{Q}=1800 \mathrm{~m}$
$\mathrm{NR} \quad \mathrm{Q}=1800 \mathrm{~m}$
$\mathrm{Q}=1000 \mathrm{~m}$
$\mathrm{Q}=700 \mathrm{~m}$
$\mathrm{Q}=1800 \mathrm{~m}$
$\mathrm{Q}=1800 \mathrm{~m}$
$\mathrm{Q}=1800 \mathrm{~m}$

\section{GRUPPO C}

6. Metrioptera caprai galvagnii (Baccetti, 1963)

9. Pholidoptera aptera goidanichi (Baccetti, 1963)

16. Podisma emiliae Ramme, 1926

22. Stenobothrus apenninus Ebner, 1915

$\begin{array}{ll}\text { SEU (APP) E } & \text { N } \\ \text { SEU (APP) E } & \text { N } \\ \text { SEU (APP) E } & \\ \text { SEU (APP) E } & \end{array}$

SEU (APP) E

SEU (APP) E
$\mathrm{Q}=1800 \mathrm{~m}$

$\mathrm{Q}=1600 \mathrm{~m}$

$\mathrm{Q}=1800 \mathrm{~m}$
$\mathrm{Q}=1600 \mathrm{~m}$
$\mathrm{NR} \quad \mathrm{Q}=1900 \mathrm{~m}$
$\mathrm{Q}=1900 \mathrm{~m}$

\section{GRUPPO D}

18. Anacridium aegyptium (Linnaeus, 1764)

AFM

NR $\quad Q=1800 \mathrm{~m}$ 
si tratta di Pholidoptera aptera goidanichi, Stenobothrus apenninus, Podisma emiliae, Metrioptera caprai galvagnii; quest'ultimo elemento è di una certa rarità, e trova nel Corno alle Scale la stazione più orientale del suo areale; è considerato a rischio di estinzione e dichiarato vulnerabile (Bardiani \& Buzzetti, 2009).

Applicando al popolamento dell'area protetta le indicazioni biogeografiche di La Greca (1996) risulta una componente angariana settentrionale (Tettigonia cantans, Bicolorana bicolor bicolor, Psophus stridulus stridulus, Stenobothrus lineatus, Stauroderus scalaris, Chorthippus parallelus parallelus) pari al $20 \%$ del popolamento totale dell'area indagata, ed una angariana meridionale (Omocestus rufipes). L'altra componente è europea s.l., mentre una piccola parte è caratterizzata da specie mediterranee, che si sono originate in fasi successive alla formazione del Mar Mediterraneo (Nemobius sylvestris, Oedipoda germanica).

Il popolamento di alta quota del Corno alle Scale, oltre il limite degli alberi $(>1600-1700 \mathrm{~m}$ in Appennino settentrionale), è da interpretare alla luce delle dinamiche già messe in evidenza da La Greca (1965, p. 31) per l'intero Appennino; le condizioni paleogeografiche della nostra penisola nel Pliocene e le notevoli mutazioni climatiche del Quaternario hanno contribuito a produrre il popolamento alticolo indagato che può essere suddiviso in due delle tre tipologie indicate da La Greca (1965):

a) neoendemiti evoluti da elementi immigrati in Ap- pennino durante le glaciazioni pleistoceniche: Stenobothrus apenninus, Podisma emiliae, Pholidoptera aptera goidanichi, Metrioptera caprai galvagnii,

b) specie immigrate verso l'Appennino nel Quaternario senza essersi differenziate in specifiche popolazioni: Bicolorana bicolor bicolor, Psophus stridulus stridulus, Omocestus viridulus, Decticus verrucivorus, Tettigonia cantans, Aeropus sibiricus sibiricus, Stauroderus scalaris.

Il popolamento ortotterologico del Parco Regionale del Corno alle Scale risulta interessante in particolare per aspetti ecologici, soprattutto di alcuni specifici elementi, per aspetti biogeografici alla luce delle interpretazioni sviluppate sull'intera catena appenninica in alcuni decenni di ricerca, infine per ragioni faunistiche consistenti nell'ampliamento della distribuzione nota di alcuni taxa.

É auspicabile una campagna di controllo per monitorare nel Parco le popolazioni di quelle specie risultate particolarmente interessanti. Pianificare la protezione di taxa quali Bohemanella frigida avrà grande importanza nella conservazione di quella che è finora nota come l'unica popolazione appenninica di questo taxon.

\section{RINGRAZIAMENTI}

Un particolare ringraziamento alla signorina Mentamer Kidanemihret (Sara) Pedroni per l'esecuzione delle fotografie agli esemplari adulti di alcune specie. 


\section{BIBLIOGRAFIA}

BACCETTI B., 1952 - Contributo alla conoscenza dell'ortotterofauna della Toscana. Ortotteri dell'Isola d'Elba. Redia, 37: 333 343.

BACCETTI B., 1954 - Note si alcuni ortotteri italiani di alta montagna. Redia, 39: pp. 361-394.

BACCETTI B., 1958 - Notulae Orthopterologicae. X. Indagini sugli Ortotteri del Gran Sasso d'Italia per il Centro di Entomologia Alpina. Redia, 43: pp. 351-450.

BACCETTI B., 1963 - Notulae Orthopterologicae. XIX Ricerche sugli Ortotteri dell'Appennino Ligure orientale per il centro di Entomologia alpina e forestale del C.N.R. Redia, 48: pp. 93-163.

BARdiani M. \& Buzzetti F.M., 2009 - Blattari e Ortotteri delle Riserve Naturali “Agoraie di Sopra e Moggetto” (Liguria, Genova) e "Guadine Pradaccio" (Emilia-Romagna, Parma). (Blattaria, Orthoptera). Bollettino dell'Associazione Romana di Entomologia, 64 (1-4): pp. 69-90.

Cerretti P., Tagliapietra A., Tisato M., Vanin S., Mason F. \& Zapparoli M., 2003 - Artropodi dell'orizzonte del faggio nell'Appennino settentrionale. Primo contributo. Conservazione Habitat Invertebrati 2. Gianluigi Arcari Editore, Mantova, pp. 256.

Chapco W. \& Litzenberger G., 2002 - A molecular phylogenetic analysis of the grasshopper genus Melanoplus Stål (Orthoptera: Acrididae). Journal of Orthoptera Research, 11(1): 1-9.

FocArILE A., 1974 - Aspetti zoogeografici del popolamento di Coleotteri (Insecta) nella Valle d'Aosta. Bulletin de la Société de la Flore Valdotaine, Aosta, 28: 5-53.

Fontana P., 2003 - Orthoptera, pp. 74-76. In: Cerretti P., Tagliapietra A., Tisato M., Vanin S., Mason F., Zapparoli M., (eds.). Artropodi dell'orizzonte del faggio nell'Appennino settentrionale. Primo contributo. Conservazione Habitat Invertebrati 2. Gianluigi Arcari Editore, Mantova, pp. 256.

Fontana P., Buzzetti F.M., Cogo A. \& Odè B., 2002 - Guida al riconoscimento e allo studio di Cavallette, Grilli, Mantidi e Insetti affini del Veneto. Blattaria, Mantodea, Isoptera, Orthoptera, Phasmatodea, Dermaptera, Embiidina. Museo Naturalistico Archeologico di Vicenza Ed., Vicenza: pp. 1-592.

Fontana P., Buzzetti F.M. \& Cogo A., 2005a - Insecta Mantodea. In: Ruffo S., Stock F. (eds), Checklist e distribuzione della fauna italiana. Memorie del Museo Civico di Storia Naturale di Verona, 2. serie, Sezione Scienze della Vita 16: pp. 135136.

Fontana P., La Greca M. \& Kleukers R., 2005b - Insecta Orthopera. In: Ruffo S., Stock F. (eds), Checklist e distribuzione della fauna italiana. Memorie del Museo Civico di Storia Naturale di Verona, 2. serie, Sezione Scienze della Vita 16: pp. 137-139.

Galvagni A., 1959 - Studio ecologico sistematico sugli Ortotteroidei dei Monti Sibillini. Memorie del Museo Civico di Storia Naturale di Verona, 7: pp. 1-76.

Galvagni A., 1971 - Ricerche sugli Ortotteroidei della Romagna e delle Marche (Italia centrale, versante adriatico). Studi Trentini di Scienze Naturali, 48(2): pp. 311-411.

Harz K., 1969 - Die orthopteren Europas. I. Junk, The Hague, 749 pp.

Harz K., 1975 - Die Orthopteren Europas. II. Junk, The Hague, pp. 938.

Harz K. \& Kaltenbach A., 1976 - Die Orthopteren Europas III. Junk, The Hague, pp. 434.

LA GreCA M., 1951 - Osservazioni sulla costituzione della fauna ortotterologica degli Appennini. Bollettino di Zoologia, 18, 4-6: pp. 197- 202

LA GRECA M., 1965 - Origine degli Ortotteri appenninici di alta quota. Rendiconti Accademia Nazionale Italiana di Entomologia. Anno XIII: pp. 19-33.

LA GRECA M., 1996 - Storia biogeografia degli Ortotteri d'Italia: origine e distribuzione (Insecta, Orthoptera). Bollettino del Museo civico di Storia naturale di Verona, 20: pp. 1-46.

Massa B., Fontana P., Buzzetti F.M., Kleukers R. \& OdÉ B., 2012 - Orthoptera. Fauna d'Italia. Vol. 48. Calderini Ed. Bologna, $600 \mathrm{pp}$.

Pedroni G., 1997 - Effetti del glacialismo appenninico sull'evoluzione dell'entomofauna. In: L'ultima glaciazione. Aspetti naturalistico-ambientali e primi insediamenti umani al Corno alle Scale. Ed. C.A.I. Emilia Romagna-Toscana, pp. 111-126.

PEDRONI G., 1998 - Nuove località per Aeropus sibiricus (Linnaeus, 1767) nelle Alpi occidentali (Insecta, Orthoptera, Acrididae). Il Naturalista Valtellinese. Atti del Museo Civico di Storia naturale di Morbegno, 9: pp. 71-72. 
SkAREAs S.D. \& Hsiung C.C. (1999) - The identity of Bohemanella frigida (Boheman) (Orthoptera: Acrididae). Notes from the Lyman Entomological Museum and Research Laboratory, 26: 1-20

Stoch F. \& Vigna Taglianti A. 2005 - I corotipi della fauna italiana. In: Ruffo S., Stoch F. (eds.). Checklist e distribuzione della fauna italiana. Memorie del Museo Civico di Storia Naturale di Verona, 2.serie, Sezione Scienze della Vita, 16: pp. 25-28.

VoIsIN J.F., 1995 - Observation sur une pullulation d'Aeropus sibiricus (Insecta Orthoptera) en Grande-Sassière (Savoie): évolution pendant les années 1987 et 1988. Travaux scientifiques du Parc national de la Vanoise, 19: 183-190.

\section{Indirizzo degli autori:}

Guido Pedroni, Parco Regionale del Corno alle Scale, via Giuseppe Mazza, 2, I, 40128 Bologna.

E-mail: guidopedroni@libero.it

Filippo Maria Buzzetti, WBA World Biodiversity Association Onlus, via Trento, 59D, I, 36071 Arzignano (VI).

E-mail: filippobuzzetti@entomon.info 